\title{
Does Hip Bone Density Differ between Paretic and Non-Paretic Sides in Hemiplegic Stroke Patients? and Its Relationship with Physical Impairment
}

\author{
Hoo Young Lee ${ }^{1,2,3,4}$, Jung Hyun Park ${ }^{1,5}$, Hyanglim Lee ${ }^{6,7}$, Tae-Woo Kim ${ }^{2,3, *}$, Seung Don Yoo ${ }^{8, *}$ \\ ${ }^{1}$ Department of Medicine, Yonsei University College of Medicine, Seoul; \\ ${ }^{2}$ TBI Rehabilitation Center, National Traffic Injury Rehabilitation Hospital, Yangpyeong; \\ ${ }^{3}$ Department of Rehabilitation Medicine, Seoul National University Hospital, Seoul University College of Medicine, Seoul; \\ ${ }^{4}$ National Traffic Injury Rehabilitation Research Institute, National Traffic Injury Rehabilitation Hospital, Yangpyeong; \\ ${ }^{5}$ Department of Rehabilitation Medicine, Rehabilitation Institute of Neuromuscular Disease, Gangnam Severance Hospital, Yonsei \\ University College of Medicine, Seoul; \\ ${ }^{6}$ Department of Internal Medicine, National Traffic Injury Rehabilitation Hospital, Yangpyeong; \\ ${ }^{7}$ Department of Internal Medicine, Seoul National University Hospital, Seoul University College of Medicine, Seoul; \\ ${ }^{8}$ Department of Physical Medicine and Rehabilitation, Kyung Hee University Hospital at Gangdong, Kyung Hee University College of \\ Medicine, Seoul, Korea
}

\section{Corresponding author}

\section{Seung Don Yoo}

Department of Physical Medicine and Rehabilitation, Kyung Hee University Hospital at Gangdong, Kyung Hee University College of Medicine, 892 Dongnam-ro, Gangdong-gu, Seoul 05278, Korea

Tel: +82-2-440-6171, Fax: +82-2-440-7260

E-mail:kidlife@khu.ac.kr

Tae-Woo Kim

TBI Rehabilitation Center, National Traffic Injury Rehabilitation Hospital, 260 Jungang-ro, Yangpyeong-eup, Yangpyeong 12564, Korea Tel: +82-31-580-5555, Fax: +82-31-580-5209 E-mail: taewookimmd@gmail.com; drcadaver@ naver.com

*Seung Don Yoo and Tae-Woo Kim contributed equally to this work and should be considered cocorresponding authors.

Received: August 27, 2020

Revised: October 21, 2020

Accepted: October 26, 2020

Copyright $@ 2020$ The Korean Society for Bone and Mineral Research

This is an Open Access article distributed under the terms of the Creative Commons Attribution Non-Commercial License (https://creativecommons.org/licenses/by-nc/4.0/) which permits unrestricted non-commercial use, distribution, and reproduction in any medium, provided the original work is properly cited.

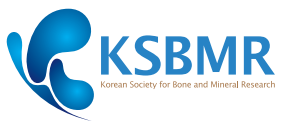

Background: Bone loss after stroke escalates the risk of fractures, mainly in the hip, leading to further disability in individuals with stroke. We aimed to investigate the skeletal effect of bone mineral density (BMD) based on the duration of onset of stroke, compare the BMD of the paretic and non-paretic sides, and elucidate the relationship between BMD and disability variables. Methods: The 31 male hemiplegic stroke patients between 20 and 70 years of age with cerebral infarction or hemorrhage were considered in this study. Subacute and chronic cases included 13 and 18 patients with lag time from the onset of 1 to 6 months and beyond 6 months, respectively. BMD in the lumbar, paretic, and non-paretic hip as well as the disability variables were analyzed retrospectively. Results: The subacute group showed a significant reduction in the femoral neck BMD on the paretic side compared to that on the non-paretic side based on T-scores $(P=0.013)$. Bone loss was significantly correlated with lower limb muscle strength and overall physical impairment $(P<0.05)$. The chronic group demonstrated significant reduction in femur neck and total femur BMD on the paretic side compared to that on the non-paretic side based on T-scores ( $P=0.002$ and $P<0.001$, respectively). T-scores of BMD in the chronic phase were not significantly associated with the clinical parameters. Conclusions: Early screening of bilateral hip BMD in the early stages after stroke, monitoring, and timely implementation of prevention strategies are important to minimize subsequent bone loss and prevent possible complications in patients who experience stroke.

Key Words: Bone density · Immobilization · Osteoporosis · Rehabilitation · Stroke

\section{INTRODUCTION}

Stroke is a leading cause of long-term disability among adults, and can result in survival with permanent functional limitations, including muscle weakness, poor balance, and frequent falls.[1] These impairments can lead to reduced activity and 
sedentary lifestyles, with further reduction in bone mineral density (BMD) but also alterations in bone geometric properties on the hemi-paretic side after stroke.[2] Unfavorable changes in both BMD and bone geometry, as well as motor, sensory and visual/perceptual deficits that predispose patients to falls have contributed to an exaggerated risk of skeletal fragility and incidence of fractures, mainly of the hip, leading to further disability and reduced survival in individuals with stroke.[3-5] The risk of a hip-joint fracture in stroke patient is 2 to 4 times higher than that of normal adults.[6] Within 12 months of stroke, human adult fracture risk is increased up to 7-fold that of age matched controls.[4]

Despite these serious sequelae of stroke, the exact mechanism of reduced BMD after stroke has not been clarified. Because stroke is a well-defined event and all genetic and most environmental factors are controlled for when the paretic and non-paretic extremities in each subject are compared, previous studies found that hemiplegia-induced bone loss is associated with paresis, reduced mobility and bone load reduction.[7-10] Endocrine factors, nutritional factors, and pharmacological factors are other known physiopathology of post-stroke osteoporosis.[11,12] Further, time since menopause in women demonstrated major association. $[3,13]$ One limitation, however, is that possible influences on BMD reduction from neural damage, were overlooked. Only limited information is available from stroke and non-stroke animal models which demonstrated the effects of brain and central nervous system regulation on bone turnover, suggesting that stroke induced neural damage may directly increase bone loss regardless of level of physical activity.[14-18]

The majority of the clinical studies that examined the bone properties in stroke patients included both sexes. Previous studies confirmed that the postmenopausal period is the strongest factor affecting the decrease of the BMD, however. To establish the optimal prevention and rehabilitation program for stroke survivors, it is important to understand the complex mechanism of neural damage on the bone loss and assess the risks. This present study aimed to investigate the skeletal effect of BMD due to onset duration of stroke, to compare the BMD of the paretic and non-paretic sides, and to relate BMD to disability variables. The primary hypothesis was that stroke would lead to a reduction in BMD in the paretic limbs particularly in the first few months after stroke onset and there would be a strong relationship between functional impairment and bone density.

Unlike women, men do not have a midlife abrupt loss of gonadal sex steroid hormone production.[19] As a result, in their middle years, they do not experience a period of accelerated bone turnover characteristic of early postmenopausal women, unless a disorder such as hypogonadism or a therapeutic castration for prostate cancer intervenes, hence, more direct correlation between the stroke and osteoporosis might be observed.[20] Therefore, to eliminate postmenopausal osteoporosis and influence of senile osteoporosis, we included only male stroke patients below 70-year-old.

\section{METHODS}

\section{Study design and participants}

The study was a retrospective, cross-sectional study of subacute and chronic male patients after stroke who were admitted to a comprehensive rehabilitation hospital in the Republic of Korea over a 4-year period from May 2016 to April 2020. The first stroke male patients with unilateral motor deficits aged from 20 to 70 years were included to eliminate postmenopausal as well as senile osteoporosis. Patients younger than 20 years or older than 70 years, acute patients who had a stroke less than 1 month previously, previous strokes affecting the sensorimotor system, terminal illness, presence of osteosynthetic material in the femoral neck, a history of hip fracture and unilateral bone diseases affecting BMD asymmetrically, such as osteosarcomas and osteomyelitis, patients with bisphosphonaterelated medication history, and secondary osteoporosis before the stroke were excluded. The first stroke was diagnosed through computed tomography or magnetic resonance imaging. The BMD was measured using dual energy X-ray absorptiometry (DXA) scan (GE lunar advance prodigy; Hologic Inc., Bedford, UK) at L1-L4, femur neck, and total proximal femur of both paretic and non-paretic lower limbs. The BMD-test results were analyzed using the T-score and absolute BMD $\left(\mathrm{g} / \mathrm{cm}^{2}\right)$.

The patients were divided into subacute stroke group (13 patients) and chronic stroke group (18 patients). Based on the patients' medical records, the baseline demographic characteristics including the age and gender, body mass index (BMl; $\mathrm{kg} / \mathrm{m}^{2}$ ) with weight and height, past history, medical history, the total serum 25 -hydroxy-vitamin $D$ 
(25[OH]D) level, and BMD-test results at admission were reviewed. As regards clinical parameters, the muscle strength of lower extremity, spasticity, balance function, ambulatory level, and activity levels of daily living were collected. Strength was determined via manual muscle testing (MMT) of bilateral ankle dorsal and plantar flexors, knee flexors and extensors, and hip flexors, extensors, adductors, and abductors, based on MMT scores ranging from 0 to 80.[21] Spasticity was assessed using the most severe values of modified Ashworth scale scores of ankle plantar flexors, knee flexors and extensors, and hip flexors, extensors, and adductors. Balance function was evaluated with the Berg Balance Scale (BBS). BBS was used for the comprehensive evaluation of patients' sitting balance, standing balance, and walking ability. BBS includes 14 common tasks of ability to maintain position or movements of increasing difficulty by decreasing the base of support from seated to standing and single-limb support. The Functional Ambulatory Category (FAC) scores were utilized as outcome measures of gait functional status. The overall physical function was assessed by the total scores of the Korean version of modified Barthel Index (K-MBI).

This study was approved by the Institutional Review Board of the National Traffic Injury Rehabilitation Hospital (No. NTRH-20001). It was conducted in accordance with the Declaration of Helsinki.

\section{Sample size and statistical analysis}

A priori sample size calculation for primary outcome was performed by adopting a previously published mean and standard deviation of decrease in BMD following stroke. [22] The minimum sample size for a error 0.05 , power 0.95 , and effect size 0.74 was calculated. Employing these inputs, 27 total patients would be necessary. Consequently, our power analysis demonstrated a power of $98 \%$.

The Mann-Whitney $U$ test was used to compare the Tscores and the absolute BMD data between the subacute and chronic stroke groups. The Wilcoxon signed rank test was used to compare the T-scores and the absolute BMD data between paretic and non-paretic hips in each group. Demographic and clinical variables, in particular incidence of osteoporosis and comorbidities, were compared between the groups using $\chi^{2}$ tests. The Spearman's rho correlations were used to investigate the presence of any correlations between the BMD-test results and other clinical parameters.
For all tests a level of significance of $P$ less than 0.05 was used. The statistical analysis was performed using SPSS version 25.0 (SPSS Inc., Chicago, IL, USA), and power analysis was performed with $G^{*}$ Power 3.1 for Windows systems.[23]

\section{RESULTS}

\section{Baseline clinical characteristics}

The baseline demographic data and clinical information

Table 1. Baseline demographics and clinical parameters of male patients with subacute and chronic stroke

\begin{tabular}{|c|c|c|c|}
\hline Characteristics & Subacute $(n=13)$ & Chronic $(n=18)$ & $P$-value \\
\hline Age (yr) & $55(47-61)$ & $57(38.75-61.25)$ & 0.679 \\
\hline BMI $\left(\mathrm{kg} / \mathrm{m}^{2}\right)$ & $23.26(21.93-27.11)$ & $23.43(23.43-25.05)$ & 0.953 \\
\hline Hemiplegia (right) & $8(61.5)$ & $12(66.7)$ & 0.828 \\
\hline Diagnosis (infarction) & $5(38.5)$ & $8(44.4)$ & 0.798 \\
\hline Duration (month) & $1.83(1.13-2.9)$ & $10.3(7.55-15.16)$ & $<0.001^{\mathrm{a}}$ \\
\hline Total MMT & $56(53.5-61.5)$ & $60.5(57-64)$ & 0.125 \\
\hline MAS & $0(0-1)$ & $1(0-1)$ & 0.133 \\
\hline BBS & $34(2.5-41)$ & $41(31.5-50)$ & $0.045^{\mathrm{a}}$ \\
\hline FAC & $1(0-2)$ & $3(2-4)$ & $0.001^{a}$ \\
\hline K-MBI & $42(34.5-65.5)$ & $69(59.5-89.25)$ & $0.004^{\mathrm{al}}$ \\
\hline Osteoporosis ${ }^{b)}$ & $0(0.0)$ & $8(44.4)$ & $0.010^{\mathrm{a}}$ \\
\hline Total serum $25(\mathrm{OH}) \mathrm{D}$ & $12.7(7.2-15.8)$ & $21.5(14.1-28.7)$ & $0.020^{\mathrm{a}}$ \\
\hline \multicolumn{4}{|l|}{ Comorbidities $^{\text {b) }}$} \\
\hline HTN & $8(61.5)$ & $7(38.9)$ & 0.285 \\
\hline DM & $7(53.8)$ & $2(11.1)$ & $0.017^{a l}$ \\
\hline CAD & $0(0.0)$ & $4(22.2)$ & 0.120 \\
\hline$A F$ & $0(0.0)$ & $2(11.1)$ & 0.497 \\
\hline \multicolumn{4}{|l|}{ Medication $^{\text {b) }}$} \\
\hline PPI & $7(53.8)$ & $6(33.3)$ & 0.460 \\
\hline Gabapentin & $2(15.4)$ & 4 (22.2) & 0.672 \\
\hline$A E D^{c l}$ & $2(15.4)$ & $5(27.8)$ & 0.581 \\
\hline SSRI/SNRI & $3(23.1)$ & $9(50.0)$ & 0.141 \\
\hline NSAID & $5(38.5)$ & $8(44.4)$ & 0.721 \\
\hline Warfarin & $0(0.0)$ & $1(5.6)$ & 1.000 \\
\hline Benzodiazepine & $0(0.0)$ & $1(5.6)$ & 1.000 \\
\hline Vitamin D & $0(0.0)$ & $2(11.1)$ & 0.492 \\
\hline
\end{tabular}

The data is presented as median (interquartile range) or number (\%). a) $P<0.05$. ${ }^{\text {bC}}$ Comparisons were made with chi-square analyses. ${ }^{\text {clPheno- }}$ barbital, phenytoin, topiramate, clonazepam.

BMI, body mass index; MMT, manual muscle testing; MAS, Modified Ashworth Scale; BBS, Berg Balance Scale; FAC, Functional Ambulatory Category; K-MBI, Korean version of modified Barthel Index; 25(OH)D, 25-hydroxy-vitamin D; HTN, hypertension; DM, diabetes mellitus; CAD, coronary artery disease; $\mathrm{AF}$, atrial fibrillation; $\mathrm{PPI}$, proton pump inhibitor; AED, anti-epileptic drug; SSRI, selective serotonin reuptake inhibitor; SNRI, serotonin-norepinephrine reuptake inhibitors; NSAID, nonsteroidal anti-inflammatory drug. 
are presented in Table 1. There were no significant differences in age, BMl, subclassification of stroke, proportion of right-sided weakness, muscle strength, spasticity, and medication history. In terms of comorbidities, diabetes mellitus (DM) was significantly higher in the subacute group compared with the chronic group $(P=0.017)$. There was no difference between the groups for other comorbidities. The chronic stroke groups demonstrated significantly advanced balance and ambulatory ability and activities of daily living ( $P=0.045, P=0.001$, and $P=0.004$, respectively). The median FAC of subacute group was 1 and chronic group was 3 . Namely, the subacute stroke patients were wheelchairbound in most of the time, and needed firm continuous support from 1 person who helps carrying weight and with balance during rehabilitative training whereas the chronic stroke patients required verbal supervision or stand-by help from one person without physical contact. The median $\mathrm{K}-\mathrm{MBI}$ score of subacute group was 42 , namely, severely dependent during activities of daily living and chronic group was 69 , or moderately dependent. Granger et al. [24] suggested $60 / 100$ as the cut-off between marked dependence and higher levels of independence. Although the chronic group demonstrated significantly better balance and ambulatory function and higher level of total serum $25(\mathrm{OH}) \mathrm{D}$ compared with the subacute group, point prevalence of osteoporosis was significantly higher in the chronic phase after the stroke.

\section{Bone density difference between paretic and non-paretic femur in subacute and chronic stroke groups}

As shown in Table 2, in the subacute phase after stroke, significant reduction in the femur neck BMD on the paretic side compared to the nonparetic side was observed $(P=0.013)$. The T-score values of the paretic femur neck and non-paretic femur neck were $-0.90(-1.70$ to -0.55$)$ and -0.70 (-1.30

Table 2. Comparison of T-scores and absolute BMD between the affected and unaffected limbs in subacute and chronic groups

\begin{tabular}{|c|c|c|c|c|c|c|c|}
\hline \multirow{2}{*}{ Group } & \multirow{2}{*}{ Outcome measures } & \multicolumn{2}{|c|}{ Femur neck } & \multirow{2}{*}{$P$-value } & \multicolumn{2}{|c|}{ Total proximal femur } & \multirow{2}{*}{$P$-value } \\
\hline & & Paretic & Non-paretic & & Paretic & Non-paretic & \\
\hline \multirow[t]{2}{*}{ Subacute } & T-scores & $-0.90(-1.70,-0.55)$ & $-0.70(-1.30,-0.40)$ & $0.013^{\mathrm{bl}}$ & $-0.40(-1.05,0.05)$ & $-0.40(-1.15,0.10)$ & 0.788 \\
\hline & Absolute BMD $\left(\mathrm{g} / \mathrm{cm}^{2}\right)$ & $0.98(0.85,1.01)$ & $0.96(0.89,1.02)$ & 0.173 & $1.06(0.93,1.10)$ & $1.04(0.95,1.10)$ & 0.133 \\
\hline \multirow[t]{2}{*}{ Chronic } & T-scores & $-1.90(-2.83,-1.10)$ & $-1.80(-2.50,-0.68)$ & $0.002^{\mathrm{bl}}$ & $-1.60(-2.78,-0.60)$ & $-1.15(-2.2,-0.35)$ & $<0.001^{\mathrm{b} b}$ \\
\hline & Absolute BMD $\left(\mathrm{g} / \mathrm{cm}^{2}\right)$ & $0.83(0.73,0.97)$ & $0.86(0.79,0.93)$ & $0.034^{\mathrm{al}}$ & $0.88(0.77,1.01)$ & $0.96(0.88,1.04)$ & $0.001^{b)}$ \\
\hline
\end{tabular}

a) $P<0.05$. b) $P<0.01$.

$\mathrm{BMD}$, bone mineral density.

Table 3. Comparison of T-scores in the lumbar spine, affected, and unaffected limbs between 2 groups

\begin{tabular}{|c|c|c|c|c|c|c|}
\hline \multirow{2}{*}{ Site } & \multicolumn{2}{|c|}{ T-score } & \multirow{2}{*}{$P$-value } & \multicolumn{2}{|c|}{ Absolute BMD $\left(\mathrm{g} / \mathrm{cm}^{2}\right)$} & \multirow{2}{*}{$P$-value } \\
\hline & Subacute group & Chronic group & & Subacute group & Chronic group & \\
\hline L1-2 & $-0.20(-1.40,0.45)$ & $-1.30(-2.52,-0.48)$ & 0.062 & $1.19(1.04,1.26)$ & $1.07(0.90,1.16)$ & 0.089 \\
\hline L1-3 & $-0.10(-1.15,0.70)$ & $-1.05(-2.38,0.00)$ & 0.082 & $1.21(1.08,1.31)$ & $1.10(0.93,1.22)$ & 0.125 \\
\hline L1-4 & $0.10(-0.95,0.80)$ & $-0.80(-2.08,0.10)$ & 0.089 & $1.25(1.11,1.34)$ & $1.19(0.98,1.25)$ & 0.183 \\
\hline L2-3 & $-0.20(-1.00,0.90)$ & $-1.20(-2.48,0.03)$ & 0.068 & $1.23(1.13,1.36)$ & $1.16(0.95,1.26)$ & 0.125 \\
\hline $\mathrm{L} 2-4$ & $0.20(-0.70,1.05)$ & $-0.70(-2.03,0.38)$ & 0.097 & $1.28(1.17,1.39)$ & $1.23(1.00,1.31)$ & 0.183 \\
\hline L3-4 & $0.30(-0.55,1.25)$ & $-0.50(-2.03,1.13)$ & 0.146 & $1.29(1.19,1.41)$ & $1.26(1.03,1.40)$ & 0.226 \\
\hline \multicolumn{7}{|l|}{ Femur neck } \\
\hline Paretic & $-0.90(-1.70,-0.55)$ & $-1.90(-2.83,-1.10)$ & $0.038^{\mathrm{a})}$ & $0.98(0.85,1.01)$ & $0.83(0.73,0.97)$ & 0.089 \\
\hline Non-paretic & $-0.70(-1.30,-0.40)$ & $-1.80(-2.50,-0.68)$ & $0.025^{\mathrm{a})}$ & $0.96(0.89,1.02)$ & $0.86(0.79,0.93)$ & 0.056 \\
\hline \multicolumn{7}{|c|}{ Total proximal femur } \\
\hline Paretic & $-0.40(-1.05,0.05)$ & $-1.60(-2.78,-0.60)$ & $0.005^{b l}$ & $1.06(0.93,1.10)$ & $0.88(0.77,1.01)$ & $0.010^{\mathrm{a})}$ \\
\hline Non-paretic & $-0.40(-1.15,0.10)$ & $-1.15(-2.2,-0.35)$ & 0.115 & $1.04(0.95,1.10)$ & $0.96(0.88,1.04)$ & 0.135 \\
\hline
\end{tabular}

a) $P<0.05$. b) $P<0.01$.

$\mathrm{BMD}$, bone mineral density. 


\section{JBM}

to -0.40), respectively. As regards T-score values of total proximal femur, significant difference was not demonstrated between the affected and unaffected sides $(P=0.788)$ in the subacute group. The T-score values of the paretic and non-paretic total proximal femur were -0.40 ( -1.05 to 0.05 ), and $-0.40(-1.15$ to 0.10$)$, respectively.

In the chronic phase after stroke, significant reduction in the femur neck as well as total proximal femur BMD on the paretic side compared to the non-paretic side was demonstrated ( $P=0.002$ and $P<0.001$, respectively). The T-score values of the paretic femur neck and non-paretic femur neck were -1.90 (-2.83 to -1.10$)$ and -1.80 ( -2.50 to -0.68$)$, respectively. And the T-score values of the paretic and nonparetic total proximal femur were $-1.60(-2.78$ to -0.60$)$ and $-1.15(-2.20$ to -0.35$)$, respectively. Further, the chronic group developed significantly more bone loss in the absolute BMD in affected femur neck as well as affected total proximal femur compared to the unaffected side ( $P=0.034$, $P=0.001$, respectively).

The chronic group showed significant decreases in T-score values for paretic femur neck, paretic total proximal femur, and also non-paretic femur neck BMD compared to the subacute group $(P<0.05)$. T-score values for the lumbar spine and non-paretic total proximal femur were unchanged in all groups (Table 3).

\section{Lumbar bone density difference between subacute and chronic stroke groups}

Results of lumbar spine BMD are shown in Table 3. Significant differences were not demonstrated in BMD values in L1-L4 and other sites. BMD measured in subacute group showed normal bone density in all sites, however, BMD measured in chronic group for L1-2, L1-3, and L2-3 indicated osteopenia, where median T-scores of $-1.30,-1.05$, and -1.20 were observed, respectively.

\section{Clinical variables associated with BMD results} The clinical parameters and the BMD results of the groups were analyzed using the Spearman correlation (Table 4, 5). The subacute group demonstrated significant correlations between the T-score results of femoral neck and the total proximal femur on the paretic side and the lower extremity MMT scores $(P<0.05)$. In the subacute group, the T-score results of the femoral neck and the total proximal femur in paretic and non-paretic femurs were significantly related

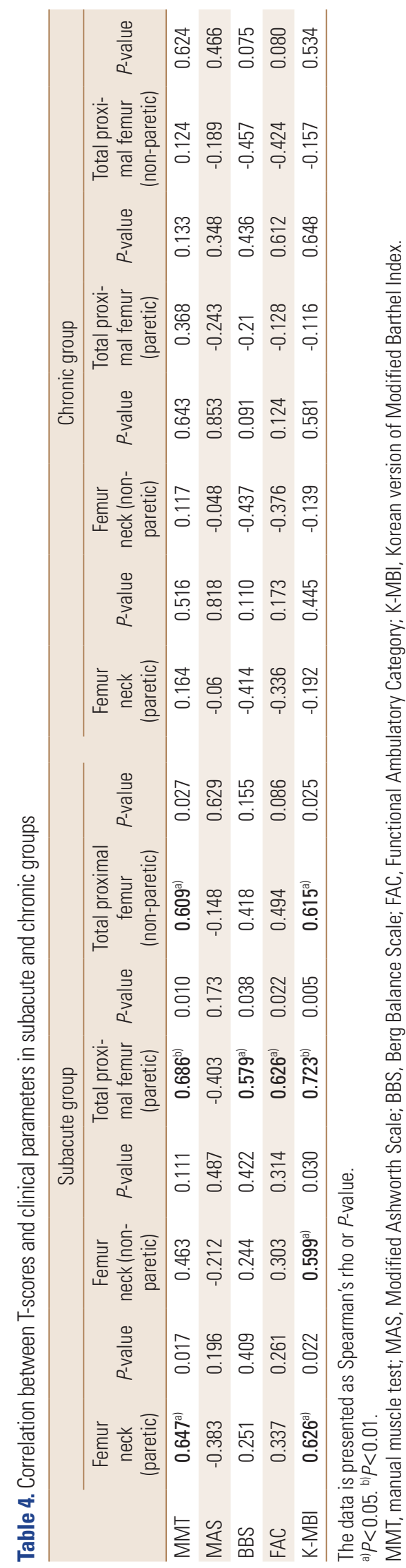

https://e-jbm.org/ 


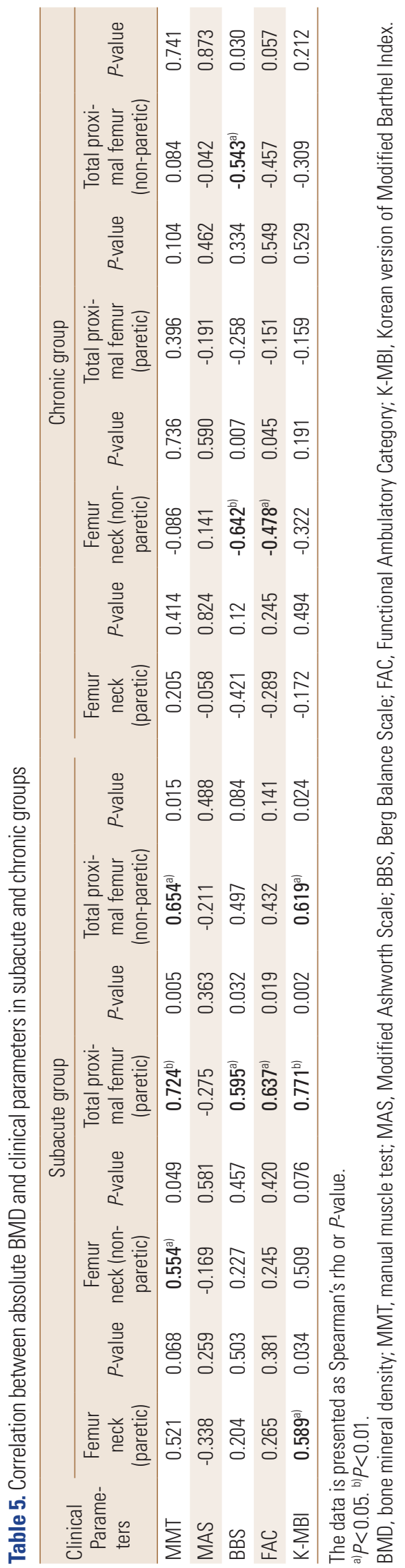

with the $\mathrm{K}-\mathrm{MBI}$ results $(P<0.05)$. These results suggest that the impaired ambulatory function during daily living reduced the BMD in both paretic and non-paretic hips and decreased muscle strength in the lower limbs significantly reduced the BMD in the paretic hip. The chronic group did not show any correlations between the clinical parameters and the BMD results.

\section{DISCUSSION}

The best practice for post-stroke recovery is intensive rehabilitation to reduce the initial impact caused by the disease, prevent complications, and maximize functional improvement.[25] Stroke is now a well-recognized risk factor for hip fracture and preventing the development of hemiosteoporosis should be a priority in the management of patients with stroke.[2] In this study, we examined the skeletal effect of BMD due to onset duration of stroke. The results showed that in the early-stroke stage, T-score identified in the femur neck was significantly lower in the affected side compared to the unaffected side. In the late-stroke stage, the affected femur neck demonstrated significantly lower T-scores in the femur neck and total proximal femur and also in the absolute BMD in the total proximal femur compared to the unaffected side.

The subacute group showed significant relationship between the T-score values of the femur neck and total proximal femur BMD on the paretic side and the muscle strength of lower limbs and overall physical impairment. The chronic group did not demonstrate any significant relationships between clinical parameters and T-scores of femoral BMD.

The current guidelines for osteoporosis diagnostics and management do not take into account post-stroke osteoporosis. Unilateral measurement of proximal femoral BMD is typically performed in patients with other forms of the disease (i.e., postmenopausal, senile or secondary), however, it may underestimate the development of bilateral difference in T-score values of femoral BMD in the early post-stroke stage. Because hip fracture after stroke leads to poorer outcomes, accurate BMD measurement is important to provide timely and optimal prevention programs. Hence, bilateral hip BMD measurement using DXA may be imperative for early screening and monitoring of post-stroke hemiosteoporosis.

Central to our understanding, hemiplegic stroke and 
subsequent immobility predispose patients to bone loss, resulting in a rapid reduction in BMD in the hemiplegic hip. $[2,7]$ Bone loss starts in the days immediately following vascular brain injury and progressively accumulates until the 3rd to 4th month after stroke.[26] Bone loss then progresses at lower speed almost until the end of the first year after stroke.[11,27]

The exact physiopathology of immobilization-induced osteopenia is not clearly understood, however. Previous studies reported the correlation between serum calcium measurements and the Barthel Index and implied that the increased calcemia resulted from bone resorption during immobilization.[12] Other relationships have been reported between degree of paresis, reduced mobility and osteoporosis.[7-10] Nevertheless, these previous investigations mainly analyzed upper limb BMD, whereas studies on the lower limb BMD is lacking. Worthen et al. [28] reported the walking correlates with bone density in chronic ambulatory stroke patient. Patients who could walk more and who had a higher vertical ground reaction force maintained a higher BMD.

In this study, the K-MBI score and FAC of the subacute group were 42 and 1 , respectively, which indicated that subjects were wheel-chair bound in most of the time, severely dependent in all aspects of daily living, and required maximal assist during gait training. On the other hand, the chronic group scored higher physical performance and walking ability compared to the subacute group. Although stroke survivors regained walking ability in the chronic phase, profound loss of BMD in the paretic hip persisted. Further, the chronic group did not demonstrate previously reported correlates of immobility, degree of paresis and bone loss.

It is worth mentioning that Jørgensen and Jacobsen [29] investigated changes in lean muscle mass and bone mineral content (BMC) of the legs during the first year after stroke according to the patient's ambulatory level. Lean body mass was lost rapidly and regained quickly, whereas BMC loss continued, especially on the paretic side.

We found that in the subacute phase after stroke, the weaker the muscle strength of lower limbs, the more the bone loss in the hemiplegic femur. This may suggest that early post-stroke BMD is related to the muscle-bone link. [2] Muscle contractions provide a rich source of mechanical loading to bone, which may, in turn, induce bone adap- tations. Functional changes in muscle characteristics such as the muscle weakness may have crucial influence on hemiplegic bone tissue. Further, in the subacute phase after stroke, the more severe the disability during the activities of daily living, the lesser the T-scores in both hemiplegic and non-hemiplegic femurs. It may indicate that the stroke induced imbalance between bone formation and degradation may directly increase bone loss in both paretic and non-paretic limbs regardless of unilateral motor deficit or local immobilization. Previous studies concerning the relationship between bone turnover and bone density in stroke patients demonstrated that biochemical markers of bone formation were reduced (when compared to controls) in the early phase of stroke recovery and throughout the first year in addition to a marked early increase in resorption markers.[11,30-32]

In this study, the chronic stroke survivors demonstrated profound loss of BMD in the paretic femur neck and further, in the total proximal femur compared to the non-paretic side. They did not show any significant correlations between reduced BMD and clinical parameters. Lack of association between level of physical impairment and bone loss in the chronic phase may indicate the skeletal effects of neural damage after stroke.

Recent studies reported the concept that the central nervous system may control bone formation and regulation of bone mass. Ducy and co-workers [33] elucidated the inhibitory effect of leptin on bone formation. Takeda et al. [34] examined the low bone mass phenotype in dopamine transporter deficient mice and tested the possible involvement of the sympathetic nervous system (SNS), which is known to be activated by leptin signaling. Bone formation and bone mass decreased after treatment with the b-adrenergic agonist isoproterenol and increased with propranolol, a b-adrenergic antagonist. These responses were resistant to leptin infusion, leading to the conclusion that SNS is the downstream mediator of leptin's central control of bone formation.[34,35] Previous studies have shown sympathetic outflow is elevated after stroke in proportion to stroke size, resulting in increased epinephrine and norepinephrine in the blood and cerebrospinal fluid of stroke patients.[36-38] Central control was also supported by increased bone synthesis and bone mass following inactivation of the $Y 2$ neuropeptide $Y$ receptor in brain.[39]

The strength of the study was that postmenopausal os- 
teoporosis as well as senile osteoporosis were eliminated. Thus, more direct correlation between the stroke and osteoporosis might be investigated. Moreover, we restricted subjects to male gender in regard to higher mortality after fractures in men, not least after a hip fracture, as well as standardized mortality in men being higher than in women who are treated for osteoporosis.[40] After 5 years, the mortality rates were $48.2 \%$ in men treated for osteoporosis and $24.6 \%$ in controls and $28.3 \%$ in women with osteoporosis and $31.9 \%$ in controls. The mortality rate at 10 years was $69.7 \%$ in men (45.4\% in controls) and $50.2 \%$ in women (50.8\% in controls).[40] Further, the investigation on BMD and clinical manifestations was focused on hip, of which the fracture is the most serious and disabling. The novelty of the methodology and the study results show that pathophysiology of post-stroke osteoporosis differs from other forms of the disease and is more evident within the paretic side. The results imply that strategies for preservation of bone mass in non-stroke populations are not automatically directly transferrable to stroke survivors. Prevention strategies for hemiosteoporosis should include exercises, early stand-up, and active rehabilitation along with pharmacological treatment. Furthermore, we elucidated the correlations of a wide range of clinical variables with the BMD in femur neck and proximal total femur on both paretic and non-paretic sides.

There are several limitations of this study that need to be addressed. The first limitation is the relatively small sample size compared to other osteoporosis studies. While the sample population in this study is limited, this sample size provides the required statistical power to address the primary aims of this study. Our results should be interpreted cautiously because the study was a retrospective study conducted in a single center. Therefore, similar to all retrospective analyses, selection bias could be present. Further, it is a cross-sectional study. Longitudinal studies are fundamental to understand changes in BMD and bone mass modifications after stroke. Unfortunately, few studies with a follow-up longer than 12 months are available.[7,41,42] Longitudinal studies regarding a long-term course of bone loss with larger sample sizes will be needed. In terms of comorbidities, DM was significantly higher in the subacute group than the chronic group. DM has been found to be associated with metabolic bone diseases, osteoporosis and lowimpact fractures, hence, the effect of DM to the reduction of BMD due to onset duration of stroke needs to be verified through further studies.[43] Moreover, BMD in poststroke patients may be affected by other factors not measured in our study, including nutritional status other than vitamin $D$ status and exposure to sunlight.

Hip fracture after stroke is an increasingly recognized problem and is associated with poorer outcomes. Guidelines for osteoporosis diagnosis and management do not include post-stroke osteoporosis, however. Prevention and rehabilitation strategies of post-stroke osteoporosis should be applied early and during the rehabilitation and post-rehabilitation phases based on the comprehensive understanding of complex mechanism of post-stroke bone metabolism and risk assessment.

\section{DECLARATIONS}

\section{Ethics approval and consent to participate}

This study conformed to the ethical guidelines of the 1975 Declaration of Helsinki and was approved by the Institutional Review Board of the National Traffic Injury Rehabilitation Hospital (No. NTRH-20001).

\section{Conflict of interest}

No potential conflict of interest relevant to this article was reported.

\section{ORCID}

Hoo Young Lee https://orcid.org/0000-0003-3846-943X

Jung Hyun Park https://orcid.org/0000-0003-3262-7476

Hyanglim Lee https://orcid.org/0000-0001-7158-7644

Tae-Woo Kim https://orcid.org/0000-0003-4017-549X

Seung Don Yoo https://orcid.org/0000-0003-4513-2560

\section{REFERENCES}

1. Khaw KT. Epidemiology of stroke. J Neurol Neurosurg Psychiatry 1996;61:333-8. http://dx.doi.org/10.1136/jnnp.61. 4.333.

2. Yang $F Z$, Jehu DAM, Ouyang $H$, et al. The impact of stroke on bone properties and muscle-bone relationship: a systematic review and meta-analysis. Osteoporos Int 2020; 31:211-24. http://dx.doi.org/10.1007/s00198-019-05175-4.

3. Carda S, Cisari C, Invernizzi M, et al. Osteoporosis after stroke: a review of the causes and potential treatments. Cerebro- 


\section{JBM}

vasc Dis 2009;28:191-200. http://dx.doi.org/10.1159/000 226578.

4. Kanis J, Oden A, Johnell O. Acute and long-term increase in fracture risk after hospitalization for stroke. Stroke 2001; 32:702-6. http://dx.doi.org/10.1161/01.str.32.3.702.

5. Dennis MS, Lo KM, McDowall M, et al. Fractures after stroke: frequency, types, and associations. Stroke 2002;33:728-34. http://dx.doi.org/10.1161/hs0302.103621.

6. Paker N, Bugdayci D, Tekdos D, et al. Relationship between bone turnover and bone density at the proximal femur in stroke patients. J Stroke Cerebrovasc Dis 2009;18:139-43. http://dx.doi.org/10.1016/j.jstrokecerebrovasdis.2008.09. 013.

7. Jørgensen $L$, Jacobsen BK, Wilsgaard $T$, et al. Walking after stroke: does it matter? Changes in bone mineral density within the first 12 months after stroke. A longitudinal study. Osteoporos Int 2000;11:381-7. http://dx.doi.org/10.1007/ s001980070103.

8. Pang MY, Eng JJ. Muscle strength is a determinant of bone mineral content in the hemiparetic upper extremity: implications for stroke rehabilitation. Bone 2005;37:103-11. http://dx.doi.org/10.1016/j.bone.2005.03.009.

9. Jørgensen $L$, Jacobsen BK. Functional status of the paretic arm affects the loss of bone mineral in the proximal humerus after stroke: a 1-year prospective study. Calcif Tissue Int 2001;68:11-5. http://dx.doi.org/10.1007/bf02684997.

10. Pang MY, Ashe MC, Eng JJ. Muscle weakness, spasticity and disuse contribute to demineralization and geometric changes in the radius following chronic stroke. Osteoporos Int 2007;18:1243-52. http://dx.doi.org/10.1007/s00198007-0372-6.

11. Sato $Y$, Kuno $H$, Kaji $M$, et al. Increased bone resorption during the first year after stroke. Stroke 1998;29:1373-7. http://dx.doi.org/10.1161/01.str.29.7.1373.

12. Sato Y, Fujimatsu Y, Kikuyama M, et al. Influence of immobilization on bone mass and bone metabolism in hemiplegic elderly patients with a long-standing stroke. J Neurol Sci 1998;156:205-10. http://dx.doi.org/10.1016/s0022510x(98)00041-0.

13. del Puente A, Pappone N, Mandes MG, et al. Determinants of bone mineral density in immobilization: a study on hemiplegic patients. Osteoporos Int 1996;6:50-4. http://dx.doi. org/10.1007/bf01626538.

14. Wong IP, Zengin $A$, Herzog $H$, et al. Central regulation of bone mass. Semin Cell Dev Biol 2008;19:452-8. http://
Hip Bone Density in Male Hemiplegic Stroke Patients

dx.doi.org/10.1016/j.semcdb.2008.08.001.

15. Vignaux G, Ndong JD, Perrien DS, et al. Inner ear vestibular signals regulate bone remodeling via the sympathetic nervous system. J Bone Miner Res 2015;30:1103-11. http:// dx.doi.org/10.1002/jbmr.2426.

16. Corr A, Smith J, Baldock P. Neuronal control of bone remodeling. Toxicol Pathol 2017;45:894-903. http://dx.doi. org/10.1177/0192623317738708.

17. Wee NK, Kulkarni RN, Horsnell $H$, et al. The brain in bone and fuel metabolism. Bone 2016;82:56-63. http://dx.doi. org/10.1016/j.bone.2015.10.020.

18. Borschmann KN, Rewell SS, Iuliano S, et al. Reduced bone formation markers, and altered trabecular and cortical bone mineral densities of non-paretic femurs observed in rats with ischemic stroke: A randomized controlled pilot study. PLoS One 2017;12:e0172889. http://dx.doi.org/10.1371/ journal.pone.0172889.

19. Gennari L, Bilezikian JP. Osteoporosis in men: pathophysiology and treatment. Curr Osteoporos Rep 2007;5:22-8. http://dx.doi.org/10.1007/bf02938619.

20. Kim HD, Kim SH, Kim DK, et al. Change of bone mineral density and relationship to clinical parameters in male stroke patients. Ann Rehabil Med 2016;40:981-8. http:// dx.doi.org/10.5535/arm.2016.40.6.981.

21. Hornby TG, Campbell DD, Kahn JH, et al. Enhanced gaitrelated improvements after therapist- versus robotic-assisted locomotor training in subjects with chronic stroke: a randomized controlled study. Stroke 2008;39:1786-92. http://dx.doi.org/10.1161/strokeaha.107.504779.

22. Lee KY, Kim SM, Lee KH, et al. The change of bone mineral density in relation to stroke duration and affected sides. J Korean Acad Rehabil Med 2010;34:650-5.

23. Faul F, Erdfelder E, Buchner A, et al. Statistical power analyses using $G^{*}$ Power 3.1: tests for correlation and regression analyses. Behav Res Methods 2009;41:1149-60. http: //dx.doi.org/10.3758/brm.41.4.1149.

24. Granger CV, Albrecht GL, Hamilton BB. Outcome of comprehensive medical rehabilitation: measurement by PULSES profile and the Barthel Index. Arch Phys Med Rehabil 1979;60:145-54.

25. Kim WS, Bae HJ, Lee HH, et al. Status of rehabilitation after ischemic stroke: A Korean nationwide study. Ann Rehabil Med 2018;42:528-35. http://dx.doi.org/10.5535/arm.2018. 42.4.528.

26. Takamoto S, Masuyama T, Nakajima M, et al. Alterations of 
bone mineral density of the femurs in hemiplegia. Calcif Tissue Int 1995;56:259-62. http://dx.doi.org/10.1007/bf00 318043.

27. Ramnemark A, Nyberg L, Lorentzon R, et al. Progressive hemiosteoporosis on the paretic side and increased bone mineral density in the nonparetic arm the first year after severe stroke. Osteoporos Int 1999;9:269-75. http://dx.doi. org/10.1007/s001980050147.

28. Worthen LC, Kim CM, Kautz SA, et al. Key characteristics of walking correlate with bone density in individuals with chronic stroke. J Rehabil Res Dev 2005;42:761-8. http:// dx.doi.org/10.1682/jrrd.2005.02.0036.

29. Jørgensen $L$, Jacobsen BK. Changes in muscle mass, fat mass, and bone mineral content in the legs after stroke: a 1 year prospective study. Bone 2001;28:655-9. http://dx. doi.org/10.1016/s8756-3282(01)00434-3.

30. Van Ouwenaller C, Uebelhart D, Chantraine A. Bone metabolism in hemiplegic patients. Scand J Rehabil Med 1989; 21:165-70.

31. Tanaka N, Sonoda S, Arita M, et al. Biochemical markers of bone turnover in hemiplegic patients. Jpn J Rehabil Med 1997;34:332-6. http://dx.doi.org/10.2490/jjrm1963.34.332.

32. Iwamoto J, Takeda T, Ichimura S. Relationships between physical activity and metacarpal cortical bone mass and bone resorption in hemiplegic patients. J Orthop Sci 2001; 6:227-33. http://dx.doi.org/10.1007/s007760100039.

33. Ducy P, Amling M, Takeda S, et al. Leptin inhibits bone formation through a hypothalamic relay: a central control of bone mass. Cell 2000;100:197-207. http://dx.doi.org/10.1016/ s0092-8674(00)81558-5.

34. Takeda S, Elefteriou F, Levasseur R, et al. Leptin regulates bone formation via the sympathetic nervous system. Cell 2002;111:305-17. http://dx.doi.org/10.1016/s0092-8674 (02)01049-8.

35. Harada S, Rodan GA. Control of osteoblast function and regulation of bone mass. Nature 2003;423:349-55. http:// dx.doi.org/10.1038/nature01660.

36. Meyer JS, Stoica E, Pascu I, et al. Catecholamine concentrations in CSF and plasma of patients with cerebral infarction and haemorrhage. Brain 1973;96:277-88. http:// dx.doi.org/10.1093/brain/96.2.277.

37. Mracsko E, Liesz A, Karcher S, et al. Differential effects of sympathetic nervous system and hypothalamic-pituitaryadrenal axis on systemic immune cells after severe experimental stroke. Brain Behav Immun 2014;41:200-9. http:// dx.doi.org/10.1016/j.bbi.2014.05.015.

38. Lechtenberg KJ, Meyer ST, Doyle JB, et al. Augmented $\beta 2$ adrenergic signaling dampens the neuroinflammatory response following ischemic stroke and increases stroke size. J Neuroinflammation 2019;16:112. http://dx.doi.org/10.1186/ s12974-019-1506-4.

39. Baldock PA, Sainsbury A, Couzens M, et al. Hypothalamic Y2 receptors regulate bone formation. J Clin Invest 2002; 109:915-21. http://dx.doi.org/10.1172/jci14588.

40. Abrahamsen B, Osmond C, Cooper C. Life expectancy in patients treated for osteoporosis: Observational cohort study using national Danish prescription data. J Bone Miner Res 2015;30:1553-9. http://dx.doi.org/10.1002/jbmr.2478.

41. Lam FM, Bui M, Yang FZ, et al. Chronic effects of stroke on hip bone density and tibial morphology: a longitudinal study. Osteoporos Int 2016;27:591-603. http://dx.doi.org/ 10.1007/s00198-015-3307-7.

42. Lazoura O, Groumas N, Antoniadou E, et al. Bone mineral density alterations in upper and lower extremities 12 months after stroke measured by peripheral quantitative computed tomography and DXA. J Clin Densitom 2008;11:511-7. http://dx.doi.org/10.1016/j.jocd.2008.05.097.

43. Wongdee K, Charoenphandhu N. Osteoporosis in diabetes mellitus: Possible cellular and molecular mechanisms. World J Diabetes 2011;2:41-8. http://dx.doi.org/10.4239/ wjd.v2.i3.41. 